PROCEEDINGS OF THE

AMERICAN MATHEMATICAL SOCIETY

Volume 138, Number 2, February 2010, Pages 577-591

S 0002-9939(09)10149-1

Article electronically published on October 14, 2009

\title{
CONTINUITY IN SEPARABLE METRIZABLE AND LINDELÖF SPACES
}

\author{
CHRIS GOOD AND SINA GREENWOOD
}

(Communicated by Jane M. Hawkins)

\begin{abstract}
Given a map $T: X \rightarrow X$ on a set $X$ we examine under what conditions there is a separable metrizable or an hereditarily Lindelöf or a Lindelöf topology on $X$ with respect to which $T$ is a continuous map. For separable metrizable and hereditarily Lindelöf, it turns out that there is such a topology precisely when the cardinality of $X$ is no greater than $\mathfrak{c}$, the cardinality of the continuum. We go on to prove that there is a Lindelöf topology on $X$ with respect to which $T$ is continuous if either $T^{\mathfrak{c}^{+}}(X)=T^{\mathfrak{c}^{+}+1}(X) \neq \emptyset$ or $T^{\alpha}(X)=$ $\emptyset$ for some $\alpha<\mathfrak{c}^{+}$, where $T^{\alpha+1}(X)=T\left(T^{\alpha}(X)\right)$ and $T^{\lambda}(X)=\bigcap_{\alpha<\lambda} T^{\alpha}(X)$ for any ordinal $\alpha$ and limit ordinal $\lambda$.
\end{abstract}

\section{INTRODUCTION}

If $T: X \rightarrow X$ is a function on a non-empty set $X$ and $\mathcal{P}$ is some topological property, then a fundamental and natural question asks whether one can endow $X$ with a topology that satisfies $\mathcal{P}$ and with respect to which $T$ is continuous.

This question can be traced back to Ellis [1, who asks whether there is a nondiscrete topology on $X$ with respect to which $T$ is continuous. De Groot and de Vries 4 provide a complete answer showing that, if $X$ is infinite, there is always a non-discrete metrizable topology on $X$ with respect to which $T$ is continuous. They go on to prove that, provided $X$ has at most $\mathfrak{c}$ many elements (where $\mathfrak{c}$ denotes the cardinality of the continuum), $X$ may be identified with a subset of the Cantor set. Moreover, in this last case, if $T$ is one-to-one, then $T$ may be taken to be a homeomorphism. They mention that, even assuming appropriate cardinality restrictions, it is impossible in general to make $X$ a compact metric space, though de Vries [11] proves that, if $T$ is a bijection, the Continuum Hypothesis is equivalent to the statement that there is a compact, metric topology on $X$ with respect to which $T$ is a homeomorphism provided $X$ has cardinality $\mathfrak{c}$.

The Banach Fixed Point Theorem implies that if $X$ is a compact metric space and $T: X \rightarrow X$ is a contraction, then $\bigcap_{n \in \mathbb{N}} T^{n}(X)=\{x\}$ for some unique fixed point $x$ of $T$. In a question related to Ellis's, de Groot asked whether there is a converse to Banach's theorem in the following sense: if $T: X \rightarrow X,|X|=\mathfrak{c}$ and $\bigcap_{n \in \mathbb{N}} T^{n}(X)=\{x\}$ for some $x$, is there a compact, metric topology on $X$ with

Received by the editors October 1, 2008 .

2000 Mathematics Subject Classification. Primary 37B99, 54A10, 54B99, 54C05, 54D20, 54D65, 54H20; Secondary 37-XX.

Key words and phrases. Abstract dynamical system, topological dynamical system, Lindelöf, separable metric, hereditarily Lindelöf.

(C)2009 American Mathematical Society 577 
respect to which $T$ is continuous? In general, for the compact metric case there is not; however, Janos [8] proves that there is a totally bounded metric topology on $X$ with respect to which $T$ is a contraction mapping and Iwanik, Janos and Smith 7] prove that there is a compact, Hausdorff topology on $X$ with respect to which $T$ is continuous, even without the restriction on the cardinality of $X$.

In 3, the continuity of arbitrary maps in compact Hausdorff spaces (see Theorem 1.3 below) and the continuity of bijections in compact metric spaces (Theorem 1.4 below) are characterized in terms of the orbit structures of the maps (see Definition 1.1 for the terminology). Iwanik [6] had earlier characterized continuity of bijections in compact Hausdorff spaces.

To state our theorems, we make the following definition.

Definition 1.1. Let $T: X \rightarrow X$. The relation $\sim$ on $X$, defined by $x \sim y$ if and only if there exist $m, n \in \mathbb{N}$ with $T^{m}(x)=T^{n}(y)$, is an equivalence relation, whose equivalence classes are the orbits of $T$.

If $O$ is an orbit of $T$, then we say that:

(1) $O$ is an $n$-cycle, for some $n \in \mathbb{N}$, if there are distinct points $x_{0}, \ldots, x_{n-1}$ in $O$ such that $T\left(x_{j-1}\right)=x_{j}$, where $j$ is taken modulo $n$;

(2) $O$ is a $\mathbb{Z}$-orbit if there are distinct points $\left\{x_{j}: j \in \mathbb{Z}\right\} \subseteq O$ such that $T\left(x_{j-1}\right)=x_{j}$ for all $j \in \mathbb{Z}$

(3) $O$ is an $\mathbb{N}$-orbit if it is neither an $n$-cycle for some $n \in \mathbb{N}$ nor a $\mathbb{Z}$-orbit.

Note that $O$ is an $\mathbb{N}$-orbit if and only if it is not a $\mathbb{Z}$-orbit and there are distinct points $\left\{x_{j}: j \in \mathbb{N}\right\} \subseteq O$ such that $T\left(x_{j}\right)=x_{j+1}$ for all $j \in \mathbb{N}$. If the set $S=\left\{x_{j}: j \in \mathbb{M}\right\}$ witnesses that $O$ is an $n$-cycle, $\mathbb{Z}$-orbit or $\mathbb{N}$-orbit, where $\mathbb{M}$ is an appropriate indexing set, then we say that $S$ is a spine for $O$. Of course, the spine of an $n$-cycle is unique, but spines of $\mathbb{N}$ - and $\mathbb{Z}$-orbits need not be unique.

Example 1.2. Consider the set

$$
Z=\left\{x_{n}: 0 \leq n \in \mathbb{Z}\right\} \cup\left\{x_{n, i}: 0>n \in \mathbb{Z}, i=0,1\right\} \cup\left\{y_{-n, k}: 1 \leq n \leq k \in \mathbb{N}\right\},
$$

and the map $T: Z \rightarrow Z$ defined by

$$
T(x)= \begin{cases}x_{n+1}, & x=x_{n}, 0 \leq n ; \\ x_{n+1, i}, & x=x_{n, i}, n<-1, i=0,1 ; \\ x_{0}, & x=x_{-1, i}, i=0,1 ; \\ y_{-n+1, k}, & x=y_{-n, k}, 1<n \leq k \in \mathbb{N} ; \\ x_{0}, & x=y_{-1, k}, k \in \mathbb{N} .\end{cases}
$$

The action of $T$ on $Z$ consists of a single $\mathbb{Z}$-orbit with two possible spines, $S_{0}$ and $S_{1}$, where $S_{i}=\left\{x_{n}: 0 \leq n \in \mathbb{Z}\right\} \cup\left\{x_{n, i}: 0>n \in \mathbb{Z}\right\}$. The rank of $x_{0}$ (see Definition 3.1) under $T$ in $Z$ is $\infty$.

The subset $N=\left\{x_{n}: 0 \leq n \in \mathbb{Z}\right\} \cup\left\{y_{-n, k}: 1 \leq n \leq k \in \mathbb{N}\right\}$ of $Z$ is closed under the action of $T$ and the action of $T \uparrow_{N}$ on $N$ consists of a single $\mathbb{N}$-orbit with infinitely many possible spines, $S_{k}^{\prime}$ for each $k \in \mathbb{N}$, where $S_{k}^{\prime}=\left\{x_{n}: 0 \leq n \in\right.$ $\mathbb{Z}\} \cup\left\{y_{-m, k}: 1 \leq m \leq k\right\}$. The rank of $x_{0}$ in $N$ under $T \uparrow_{N}$ is $\omega$.

Theorem $1.3([3])$. Let $T: X \rightarrow X$. There is a compact, Hausdorff topology on $X$ with respect to which $T$ is continuous if and only if

$$
T\left(\bigcap_{m \in \mathbb{N}} T^{m}(X)\right)=\bigcap_{m \in \mathbb{N}} T^{m}(X) \neq \emptyset
$$


and either:

(1) $T$ has, in total, at least continuum many $\mathbb{Z}$-orbits or cycles; or

(2) $T$ has both a $\mathbb{Z}$-orbit and a cycle; or

(3) there are $n_{i} \in \mathbb{N}, i \leq k<\infty$, such that $T$ has an $n_{i}$-cycle for each $i$ and, whenever $T$ has an $n$-cycle, $n$ is divisible by $n_{i}$, for some $i \leq k$; or

(4) the restriction of $T$ to $\bigcap_{m \in \mathbb{N}} T^{m}(X)$ is not one-to-one.

Theorem $1.4([3])$. Let $T: X \rightarrow X$ be a bijection. There is a compact metrizable topology on $X$ with respect to which $T$ is a homeomorphism if and only if one of the following hold:

(1) $X$ is finite.

(2) $X$ is countably infinite and either:

(a) $T$ has both a $\mathbb{Z}$-orbit and a cycle or

(b) there are $n_{i} \in \mathbb{N}, i \leq k<\infty$, such that $T$ has an $n_{i}$-cycle for each $i$ and, whenever $T$ has an $n$-cycle, $n$ is divisible by $n_{i}$, for some $i \leq k$.

(3) $X$ has the cardinality of the continuum and each of the number of $\mathbb{Z}$-orbits and the number of $n$-cycles, for $n \in \mathbb{N}$, is finite, countably infinite, or has the cardinality of the continuum.

In this paper, we address this question of continuity in Tychonoff, Lindelöf or hereditarily Lindelöf spaces. To state these results we need a further definition.

Definition 1.5. Let $T: X \rightarrow X$ be a function. For any $A \subseteq X$, any ordinal $\alpha$ and any limit ordinal $\lambda$, define $T^{\alpha+1}(A)=T\left(T^{\alpha}(A)\right)$ and $T^{\lambda}(A)=\bigcap_{\alpha \in \lambda} T^{\alpha}(A)$.

We prove the following.

Theorem 1.6. Let $T: X \rightarrow X$ be a function. There is a (zero-dimensional) Tychonoff, Lindelöf topology on $X$ with respect to which $T$ is continuous provided either

(1) $T^{\mathfrak{c}^{+}}(X)=T^{\mathfrak{c}^{+}+1}(X) \neq \emptyset$ or

(2) $T^{\alpha}(X)=\emptyset$ for some $\alpha<\mathfrak{c}^{+}$.

Corollary 1.7. Let $T: X \rightarrow X$ be a function. There is a (zero-dimensional) Tychonoff Lindelöf topology on $X$ with respect to which $T$ is continuous provided any of the following holds:

(1) $T$ is a surjection,

(2) $T$ is an injection,

(3) $T$ is a $<$-to-one function,

(4) $T$ is $a \leq \mathfrak{c}$-to-one function with at least one $\mathbb{Z}$-orbit or $n$-cycle.

Theorem 1.8. Let $T: X \rightarrow X$. The following are equivalent:

(1) $|X| \leq \mathfrak{c}$.

(2) $X$ can be identified with a subset of the Cantor set in such a way that the action of $T$ is continuous.

(3) There is a (zero-dimensional) Hausdorff, hereditarily Lindelöf topology on $X$ with respect to which $T$ is continuous.

(4) There is a (zero-dimensional) first countable, Hausdorff, Lindelöf topology on $X$ with respect to which $T$ is continuous.

(5) There is a (zero-dimensional) first countable, Hausdorff, separable topology on $X$ with respect to which $T$ is continuous. 
(6) There is a (zero-dimensional) separable metrizable topology on $X$ with respect to which $T$ is continuous.

(7) There is a continuous function $t$ from the Hilbert cube $[0,1]^{\mathbb{N}}$ to itself and an identification of $X$ with a (zero-dimensional) subset of $[0,1]^{\mathbb{N}}$ such that $T$ is the restriction of $t$ to $X$.

We are left with the question:

Question 1. Is there a map $T$ on a set $X$ that is not continuous with respect to any Lindelöf topology on $X$ ?

We conjecture that the answer is yes. In light of Theorem [1.6, we are really asking the following.

Question 2. Suppose that $T^{\mathfrak{c}^{+}}(X) \neq T^{\mathfrak{c}^{+}+1}(X)$. Is there a Tychonoff, Lindelöf topology on $X$ with respect to which $T$ is continuous?

Question 3. Suppose that $\|p\|=\mathfrak{c}^{+}$(see Definition 3.1). Is there a Lindelöf topology on $T^{-k}(p)$, for each $k>0$, such that the restriction of $T$ from $T^{-(k+1)}(p)$ to $T^{-k}(p)$ is continuous?

Question 4. Suppose that $T^{\mathfrak{c}^{+}}(X)=\emptyset$ but that $T^{\alpha}(X) \neq \emptyset$ for any $\alpha \in \mathfrak{c}^{+}$. Is there a Lindelöf topology on $X$ with respect to which $T$ is continuous?

Our notation and terminology are standard as found in [2] and [10]. The paper is organized as follows. In Section 2 we prove Theorem 1.8. The proof that Theorem 1.8 (11) implies Theorem 1.8 (17) does in fact follow from a careful reading of the construction of the Lindelöf topology in the proof of Theorem 1.6, but the argument given in this section is far more direct and geometric. The proof of Theorem 1.6 is somewhat involved, although we have taken some pains to simplify the exposition as far as possible. In Section 3 we discuss a natural tree structure on $\bigcup_{0 \leq k} T^{-k}(x)$, for each $x \in X$, and an associated notion of rank. In Section 4, we use this tree structure and the rank of points of $X$ to put as appropriate topology on each orbit. We know that, for Lindelöf $X$ and continuous $T$, the set $T^{-k}(x)$ is Lindelöf for any $x \in X$ and $0 \leq k$. So, in constructing the topology on $T^{-(k+1)}(x)$ from the topology on $T^{-k}(x)$, we use our notion of rank to keep track of which points should act as limit points, ensuring both continuity and the Lindelöf property. In the final section, we topologize $X$ by considering the various combinations of orbits, thus completing the proof of Theorem [1.6.

\section{Continuity in Separable metrizable And HEREDITARILY LINDELÖF SPACES}

De Groot (see [5]) proved that every hereditarily Lindelöf space has cardinality at most $\mathfrak{c}$. It turns out that this is the only condition required for there to be a hereditarily Lindelöf topology making a given self-map on a set continuous. A version of the proof of (10) implies (5) in Theorem 1.8 essentially follows from the proof of case (1) of Theorem 1.6, but the following argument is more natural.

Proof of Theorem 1.8. Clearly (7) implies (6) and (6) implies each of (3), (4), and (5). Arhangel'skii proved that first countable, Hausdorff Lindelöf spaces have cardinality at most $\mathfrak{c}$, Pospíšil proved that every first countable, separable (indeed density $\leq \mathfrak{c})$ Hausdorff space has cardinality at most $\mathfrak{c}$, and de Groot proved that Hausdorff 
hereditarily Lindelöf spaces also have cardinality at most $\mathfrak{c}$ (see [5]). Hence (3), (4) and (5) all imply (11). That (2) is equivalent to (11) is due to de Groot and de Vries [4. It remains to show that (11) implies (7).

We will show that, if $|X| \leq \mathfrak{c}$, then there is a continuous map $t$ on the Hilbert cube and an identification of $X$ with a zero-dimensional subset of the Hilbert cube so that $T$ corresponds to the restriction of $t$ to $X$.

Assume, then, that $T: X \rightarrow X$ and that $|X| \leq \mathfrak{c}$. Let $I=[0,1]$, let $\mathbb{H}$ denote the Hilbert cube $[0,1]^{\mathbb{N}}$, and let $S_{1}$ denote the unit circle in the plane. Since we are only trying to embed $X$ as a subspace of $\mathbb{H}$, we may assume that, for each $x \in X, T^{-1}(x)$ has cardinality $\mathfrak{c}$ (otherwise we can consider the restriction of $T$ to a subset). In particular, this means that, without loss of generality, there are no $\mathbb{N}$-orbits in $T$. It therefore suffices to construct a continuous function $t: \mathbb{H} \rightarrow \mathbb{H}$ which has $\mathfrak{c}$ many $\mathbb{Z}$-orbits and $\mathfrak{c}$ many $n$-cycles, for each $n \in \mathbb{N}$, with the property that $T^{-1}(x)$ has cardinality $\mathfrak{c}$ for each point $x$ in each of these orbits. One can then easily choose a zero-dimensional subset on which the restriction of the action of $t$ corresponds to the action of $T$.

Let $\phi$ be the homeomorphism from the cylinder $S_{1} \times[0,1]$ to itself defined by $\phi(\theta, x)=(\theta+2 \pi x, x)$. It is a standard fact that the orbit of the point $(\theta, x)$ is a $\mathbb{Z}$-orbit precisely when $x$ is an irrational number and is an $n$-cycle when $x=m / n$, expressed in lowest terms. Let $\mathbb{H}^{\prime}=S_{1} \times I \times \mathbb{H}$ and let $t^{\prime}: \mathbb{H}^{\prime} \rightarrow \mathbb{H}^{\prime}$ be the map defined by

$$
t^{\prime}\left(\theta, x, r_{1}, r_{2}, r_{3}, \ldots\right)=\left(\theta+2 \pi x, x, r_{2}, r_{3}, \ldots\right) .
$$

Given the continuity of $\phi$ and the shift map $\left(r_{1}, r_{2}, r_{3}, \ldots\right) \mapsto\left(r_{2}, r_{3}, \ldots\right)$ on $\mathbb{H}, t^{\prime}$ is easily seen to be a continuous map on $\mathbb{H}^{\prime}$. For any $x \in[0,1]$, consider the subset

$$
\mathbb{H}_{x}=S_{1} \times\{x\} \times\{(0,0,0, \ldots)\} \cup \bigcup_{1 \leq m} S_{1} \times\{x\} \times I^{m} \times\{(0,0,0, \ldots)\}
$$

$\mathbb{H}_{x}$ is invariant under the action of $t^{\prime}$. For each point $p$ of $\mathbb{H}_{x}, T^{-1}(p)$ has cardinality $\mathfrak{c}$ and $p$ is eventually mapped to a point of the form $(\theta, x, 0,0, \ldots)$, so the orbit of $p$ is a $\mathbb{Z}$-orbit, if $x$ is irrational, or a cycle, if $x$ is rational. Moreover, there are $\mathfrak{c}$ many such orbits in $\mathbb{T}_{x}$. It follows that (for example) the subset $\mathbb{H}^{\prime \prime}=\mathbb{H}_{\sqrt{2}} \cup \bigcup_{1 \leq n} \mathbb{H}_{1 / n}$ of $\mathbb{H}^{\prime}$ is closed under $t^{\prime}$, consists of $\mathfrak{c}$ many $\mathbb{Z}$-orbits and $\mathfrak{c}$ many $n$-cycles, for each $n \in \mathbb{N}$, and has the property that $t^{\prime-1}(p)$ has cardinality $\mathfrak{c}$ for each $p \in \mathbb{H}^{\prime \prime}$. Since $\mathbb{H}^{\prime \prime}$ is a subspace of $\mathbb{H}^{\prime}$, which is homeomorphic to $\mathbb{H}$, we are done.

\section{SELF-MAPS AND WELL-FOUNDED TREES}

In this section, we describe a natural ordinal invariant, the rank of $x \in X$ under $T$, for points under the action of $T$. This rank corresponds to the rank of wellfounded trees from descriptive set theory (see, for example, 9]), and we use it in Section 4 to index our construction of a Lindelöf topology. Our idea is, roughly, that we will only declare a point $x$, say, to be a limit point of a set of points $A$ if the rank of each $y \in A$ is no greater than the rank of $x$. This will ensure, via Lemma 3.2, that there are 'enough' points in $T^{-1}(x)$ to act as limit points for $T^{-1}(A)$.

For notational convenience, we let $T^{0}(p)=p$ or $\{p\}$ depending on the context. 
Definition 3.1. Suppose that $T: X \rightarrow X$ is a function. The rank of $x \in X$ under $T$ is

$$
\|x\|= \begin{cases}\alpha & \text { if } x \in T^{\alpha}(X) \backslash T^{\alpha+1}(X), \\ \infty & \text { if } x \in \bigcap_{\alpha \in O n} T^{\alpha}(X) .\end{cases}
$$

For each $x \in X$, and each $y, z \in \bigcup_{0 \leq k} T^{-k}(x)$, define $y \triangleleft_{x} z$ if and only if $T^{j}(z)=y$ for some $j>0$.

For each $x \in X,\left(\bigcup_{0<k} T^{-k}(x), \triangleleft_{x}\right)$ forms a well-founded tree of height $\|x\|$ if and only if $\|x\|<\infty$ (see [9, 25.5] for more on well-founded trees). For our purposes it is sufficient to see that, if $\|x\|<\|y\|$, then there is an order-preserving map from $\bigcup_{0 \leq k} T^{-k}(x)$ to $\bigcup_{0 \leq k} T^{-k}(y)$.

Lemma 3.2. Let $T: X \rightarrow X$ and let $x \in X$.

(1) $\|x\|=\infty$ if and only if there exists a sequence $x_{n}, n=0,1,2, \ldots$, such that $x_{0}=x$ and $T\left(x_{n+1}\right)=x_{n}$. In particular, if $x$ is a point on the spine of a $\mathbb{Z}$-orbit or of an $n$-cycle, then $\|x\|=\infty$, and if $\|x\|=\infty$, then $x$ is not in an $\mathbb{N}$-orbit.

(2) If $\|x\| \leq\|y\|$, then there is an order-preserving map

$$
f_{x y}:\left(\bigcup_{0 \leq k} T^{-k}(x), \triangleleft_{x}\right) \rightarrow\left(\bigcup_{0 \leq k} T^{-k}(y), \triangleleft_{y}\right)
$$

such that $f_{x y}\left(T^{-k}(x)\right) \subseteq T^{-k}(y)$ for all $k \in \mathbb{N}$.

Proof. Clearly, if there is a sequence of points $x_{n}, n \in \mathbb{N}$, such that $x_{0}=x$ and $T\left(x_{n+1}\right)=x_{n}$, then $x_{0} \in T^{\alpha}(X)$ for all ordinals $\alpha$, and so $\|x\|=\infty$. Suppose, then, that $\|x\|=\infty$, i.e. that $x \in T^{\alpha}(X)$ for all ordinals $\alpha$. If $\|y\|<\infty$ for each $y \in T^{-1}(x)$, then $\|x\|=\sup \left\{\|y\|+1: y \in T^{-1}(y)\right\}<\infty$, so there is some $x_{1} \in T^{-1}(x)$ with $\left\|x_{1}\right\|=\infty$. It follows that there is an infinite sequence $x_{n}$ with $x_{0}=x$, and $x_{n}=T\left(x_{n+1}\right)$. Hence (1) follows.

For (2), following [9, 25.6]: If $\|y\|=\infty$, then by (1), there is some sequence of points $y_{k}, k=0,1,2, \ldots$, such that $y_{0}=y$ and $T\left(y_{k+1}\right)=y_{k}$. In this case, define $f_{x y}(z)=y_{k}$ if and only if $z \in T^{-k}(x)$. If $\|y\|<\infty$, then we argue by induction. If $z \in T^{-1}(x)$, then $\|z\|<\|x\| \leq\|y\|$, so that there is some $y_{z} \in T^{-1}(y)$ such that $\|z\| \leq\left\|y_{z}\right\|$. Let $f_{z}$ be an order-preserving map from $\left(\bigcup_{0 \leq k} T^{-k}(z), \triangleleft_{z}\right)$ to $\left(\bigcup_{0 \leq k} T^{-k}\left(y_{z}\right), \triangleleft_{z}\right)$ such that $f_{z}\left(T^{-k}(z)\right) \subseteq T^{-k}\left(y_{z}\right)$ for all $k \in \mathbb{N}$. Define

$$
f_{x y}(w)= \begin{cases}y & \text { if } w=x \\ f_{z}(w) & \text { if } w \in \bigcup_{0 \leq k} T^{-k}(z), \text { for some } z \in T^{-1}(x) .\end{cases}
$$

Since $\bigcup_{0 \leq k} T^{-k}(z) \cap \bigcup_{0 \leq k} T^{-k}\left(z^{\prime}\right)=\emptyset$ for any distinct $z$ and $z^{\prime}$ in $T^{-1}(x), f_{x y}$ is well-defined and we are done.

The following rather technical looking lemma collects together a number of facts we will need later. The proof follows fairly directly from the definitions.

Lemma 3.3. Suppose that $T: X \rightarrow X$ is a function.

(1) The following are equivalent:

(a) $T^{\mathfrak{c}^{+}}(X)=T^{\mathfrak{c}^{+}+1}(X)$;

(b) for all $x \in X$, either $\|x\|<\mathfrak{c}^{+}$or $\|x\|=\infty$;

(c) for all $x \in X$, there is a subset $D_{x} \subseteq T^{-1}(x)$ such that 
(i) $\left|D_{x}\right| \leq \mathfrak{c}$ and

(ii) for each $z \in T^{-1}(x)$ there is some $y_{z} \in D_{x}$ such that $\|z\| \leq$ $\left\|y_{z}\right\|$.

(2) Let $T^{\mathfrak{c}^{+}}(X)=T^{\mathfrak{c}^{+}+1}(X)$. Let $x \in X$ and let $D_{x}$ be a subset of $T^{-1}(x)$ satisfying (1c). Suppose that $y \in D_{x}$ and that $D_{x}-\{y\}$ does not satisfy (1c). Then either

(a) $\|y\|=\infty$, so that $\|x\|=\infty$, or

(b) there is a subset $D_{x}^{\prime}$ of $T^{-1}(x)$, which does not contain $y$ but does satisfy (1c).

(3) If $T^{\mathfrak{c}^{+}}(X)=T^{\mathfrak{c}^{+}+1}(X) \neq \emptyset$, then $X$ has a $\mathbb{Z}$-orbit or an $n$-cycle for some $n \in \mathbb{N}$.

(4) If $T^{\alpha}(X)=\emptyset$ for any ordinal $\alpha$, then $X$ consists solely of $\mathbb{N}$-orbits.

Proof. For (1): To see that (a) implies (b), suppose that $T^{\mathfrak{c}^{+}}(X)=T^{\mathfrak{c}^{+}+1}(X)$ but that some $x \in X$ has $\mathfrak{c}^{+} \leq\|x\|<\infty$. Then, without loss of generality, $\|x\|=\mathfrak{c}^{+}$(if not, then some point of $\bigcup_{0 \leq n} T^{-n}(x)$ has rank $\mathfrak{c}^{+}$). But then $x \in$ $T^{\mathfrak{c}^{+}}(X)-T^{\mathfrak{c}^{+}+1}(X)$, which is a contradiction.

Suppose that (b) holds. If $\|x\|=\infty$, then there is some $y \in T^{-1}(x)$ such that $\|y\|=\infty$, so that we can let $D_{x}=\{y\}$. On the other hand, $\|x\|<\mathfrak{c}^{+}$. Then the set of ordinals $\left\{\|y\|: y \in T^{-1}(x)\right\}$ has cardinality $\leq \mathfrak{c}$, and we see that (c) holds by Lemma 3.2

To see that (a) follows from (c), suppose that $T^{\mathfrak{c}^{+}}(X) \neq T^{\mathfrak{c}^{+}+1}(X)$, so that there is a point $x$ such that $\|x\|=\mathfrak{c}^{+}$, in which case $\sup \left\{\|y\|: y \in T^{-1}(x)\right\}=\mathfrak{c}^{+}$. Since $\mathfrak{c}^{+}$has cofinality strictly greater than $\mathfrak{c}$, no such subset $D_{x}$ can exist.

For (2): Suppose that $D_{x}$ and $y$ are as stated and suppose that $\|y\| \neq \infty$, so that $\|y\|<\mathfrak{c}^{+}$. Note that $T^{-1}(x) \neq\{y\}$, since otherwise $D_{x}-\{y\}=\emptyset$ vacuously satisfies (1c). Let $Z$ be the set of all $z \in T^{-1}(x)$ for which $\|z\|>\left\|z^{\prime}\right\|$ for all $z^{\prime} \in D_{x}-\{y\}$. $Z$ is non-empty, since otherwise $D_{x}-\{y\}$ would satisfy $(1 \mathrm{c})$, and $\left(D_{x}-\{y\}\right) \cup Z$ satisfies (1c) (ii). Clearly, for each $z \in Z,\|z\| \leq\|y\|$. Since $\|y\|<\mathfrak{c}^{+}$, $\|y\|$ has cofinality at most $\mathfrak{c}$. But then there is a subset $Z^{\prime}$ of $Z$ of cardinality at most $\mathfrak{c}$ with the property that for all $z \in Z$ there is some $z^{\prime} \in Z^{\prime}$ such that $\|z\| \leq\left\|z^{\prime}\right\| \leq\|y\|$. Setting $D_{x}^{\prime}=\left(D_{x} \cup Z^{\prime}\right)-\{y\}$, we are done.

(3) and (4) follow from (1) of Lemma 3.2. since if $T^{\mathfrak{c}^{+}}(X)=T^{\mathfrak{c}^{+}+1}(X) \neq \emptyset$, $\|x\|=\infty$ for every $x \in T^{\mathfrak{c}^{+}}(X)$.

\section{Putting a topology on $\bigcup_{k \in \mathbb{N}} T^{-k}(p)$}

Let $\mathbb{C}$ denote a Cantor set in $[0,1]$, and for this section let us say that $(\tau, \prec)$ is an augmented graph provided:

(1) $\tau$ has a unique top element $t_{\tau}$;

(2) $m_{\tau}=\max \{m$ : there is a branch in $\tau$ of length $m\}<\infty$;

(3) there are $n_{\tau} \in \mathbb{N}$ and $n(t) \in \mathbb{N}$, for each $t \in \tau$, such that:

(a) $0<n(t) \leq n_{\tau}$;

(b) if $s \prec t$, then $n(t) \leq n(s)$;

(c) for each $s \prec t$, there are surjective projections $\pi_{s t}: \mathbb{C}^{n(s)} \rightarrow \mathbb{C}^{n(t)}$ satisfying $\pi_{r t}=\pi_{s t} \circ \pi_{r s}$, whenever $r \prec s \prec t$.

For any $F \subseteq \tau$, let $\downarrow F=\{s \in \tau: s \preceq t$ for some $t \in F\}$. 
Let $Z_{\tau}=\bigcup_{t \in \tau} \mathbb{C}^{n(t)} \times\{t\}$. For each $s \in \tau$, let $B_{s}=\bigcup_{t \preceq s} \mathbb{C}^{n(t)} \times\{t\}$. For $\bar{r} \in \mathbb{C}^{n}$ and $j \in \mathbb{N}$, let $B_{j}(\bar{r})$ denote the $1 / 2^{j}$-ball about $\bar{r}$. For any $(\bar{r}, t) \in \mathbb{C}^{n(t)} \times\{t\}$, $j \in \mathbb{N}$ and finite $F \subseteq\{s \in \tau: s \prec t\}$, let

$$
B(\bar{r}, t, j, F)=\left(\left(B_{j}(\bar{r}) \times\{t\}\right) \cup \bigcup_{s \prec t} \pi_{s t}^{-1}\left(B_{j}(\bar{r})\right) \times\{s\}\right) \backslash\left(\bigcup_{s \in F} B_{s}\right) .
$$

Let $\mathcal{T}_{\tau}$ be the topology on $Z_{\tau}$ that is generated by the collection of all such sets $B(\bar{r}, t, j, F)$.

Lemma 4.1. $\left(Z_{\tau}, \mathcal{T}_{\tau}\right)$ is a compact, zero-dimensional space. Moreover, if $Y$ is a subset of $Z_{\tau}$ with the property that for every $(\bar{r}, s) \in Y$ and $s \prec t, \pi_{s t}(\bar{r}, s) \in Y$, then $Y$ is Lindelöf.

Proof. Zero-dimensionality follows from the fact that each $B_{j}(\bar{r})$ is a clopen set.

For each $t \in \tau$, let $\rho(t)$ denote the length of the longest branch below $t$, so that $\rho(t) \leq \rho\left(t_{\tau}\right)=m_{\tau}$. To see that $Z_{\tau}$ is compact, note first that each subspace $\mathbb{C}^{n(t)} \times\{t\}$ is homeomorphic to the usual Euclidean space $\mathbb{C}^{n(t)}$. In particular, for any open cover of $Z_{\tau}$ by basic open subsets, there is a finite subcover of $\mathbb{C}^{n\left(t_{\tau}\right)} \times\left\{t_{\tau}\right\}$, $\left\{B\left(\bar{r}_{i}, t_{\tau}, j, F_{i}\right): i \leq m\right\}$. This cover must cover all of $Z_{\tau}$ except for, possibly, the sets $B_{s}, s \in F_{i}$ for some $i \leq m$. Since each $\mathbb{C}^{n(s)} \times\{s\}$ is compact and $\rho(s)<\rho\left(t_{\tau}\right)$, we may repeat this argument a finite number of times to obtain a finite subcover of $Z_{\tau}$.

Suppose then that $Y$ is a subspace of $Z_{\tau}$ with the property that whenever $(\bar{r}, s) \in$ $Y$ and $s \prec t$, then $\left(\pi_{s t}(\bar{r}), t\right) \in Y$. Note that each subset $\mathbb{C}^{n(t)} \times\{t\}$ of $Z_{\tau}$ is hereditarily Lindelöf. Let $\mathcal{U}$ be a cover of $Y$ and let $Y_{t}=Y \cap\left(\mathbb{C}^{n(t)} \times\{t\}\right)$ for each $t \in \tau$.

Since $Y_{t_{\tau}}$ is Lindelöf, it has a countable cover $\left\{U_{t_{\tau}, i}: i \in \mathbb{N}\right\} \subseteq \mathcal{U}$. Since $\left(\pi_{s t_{\tau}}(\bar{r}), t_{\tau}\right)$ is in $Y_{t_{\tau}}$ whenever $(\bar{r}, s) \in Y,\left\{U_{t_{\tau}, i}: i \in \mathbb{N}\right\}$ covers all but countably many of the sets $Y_{t}$. Let $T_{0}=\left\{t_{\tau}\right\}$ and define $T_{1}$ by letting $t \in T_{1}$ if and only if $Y_{t}$ is not covered by $\left\{U_{i}: i \in \mathbb{N}\right\}$. By the definition of the topology on $Z_{\tau}$, if $t \in T_{1}$, then $t \prec t_{\tau}$.

As for $Y_{t_{\tau}}$, for each $t \in T_{1}$, there is a countable cover $\left\{U_{t, i}: i \in \mathbb{N}\right\} \subseteq \mathcal{U}$ of $Y_{t}$. Since $\left(\pi_{s t}(\bar{r}), t\right) \in Y$, whenever $(\bar{r}, s) \in Y$ and $s \prec t,\left\{U_{t, i}: i \in \mathbb{N}\right\}$ covers all but countably many $Y_{s}$ for which $s \prec t$. Define $T_{2}$ by letting $s \in T_{2}$ if and only if $Y_{s}$ is not covered by the countable collection of open sets $\left\{U_{t, i}, i \in \mathbb{N}, t \in T_{0} \cup T_{1}\right\}$, so that $T_{2}$ is a countable set.

Repeating this argument, we obtain a series of countable sets $T_{j}$ and countable collections $\left\{U_{t, i}: i \in \mathbb{N}\right\} \subseteq \mathcal{U}$, for each $t \in T_{j}$ such that $\left\{U_{t, i}: i \in \mathbb{N}, t \in T_{0} \cup \cdots \cup T_{j}\right\}$ covers all of $Y$ except for, possibly, $\bigcup_{s \in T_{j+1}} Y_{s}$. By construction, if $s \in T_{j+1}$, then $s \prec t$ for some $t \in T_{j}$. Since the maximum length of each path through $\tau$ is $m_{\tau}$, $T_{m_{\tau}}$ is empty and, therefore, $\left\{U_{t, i}: i \in \mathbb{N}, t \in \bigcup_{j \leq m_{\tau}} T_{j}\right\}$ is a countable subcover of $\mathcal{U}$.

Now suppose that $p \in X$ is a non-spine point $p$ such that $T(p)$ is on a spine. We will identify $T^{-k}(p)$ with a Lindelöf subset of $Z_{\tau}$, for some augmented graph $\tau$, so that the action of $T$ from $T^{-k}(p)$ to $T^{-k+1}(p)$ is continuous. This, then, provides a topology on $\bigcup_{0 \leq n} T^{-n}(p)$ for each such non-spine point $p$. In Section [5] we use these topologies to put a Lindelöf topology on the whole of $X$. 
Lemma 4.2. Let $T: X \rightarrow X$ and let $O$ be an orbit of $T$ with spine $S$. Let $s \in S$ and let $p \in T^{-1}(s)-S$. Suppose that for every $x \in \bigcup_{0 \leq k} T^{-k}(p)$ there is a subset $D_{x} \subseteq T^{-1}(x)$ such that

(1) $\left|D_{x}\right| \leq \mathfrak{c}$ and

(2) for each $z \in T^{-1}(x)$, there is some $y_{x z} \in D_{x}$ such that $\|z\| \leq\left\|y_{x z}\right\|$.

Then for each $k \geq 0$ there is a (zero-dimensional) Tychonoff Lindelöf topology $\mathcal{T}_{k}$ on $T^{-k}(p)$ with respect to which the action of $T$ from $T^{-k-1}(p)$ to $T^{-k}(p)$ is continuous.

Proof. For each $k \geq 0$, we will embed $T^{-k}(p)$ as a subset of $Z_{\tau_{k}}$ for some augmented graph $\left(\tau_{k}, \prec_{k}\right)$. To simplify the notation, once it has been embedded, we will often refer to $T^{-k}(p)$ as a subset of $Z_{\tau_{k}}$, referring to points of $T^{-k}(p)$ as points of $Z_{\tau_{k}}$. We will further ensure that for any $x=(\bar{r}, s) \in T^{-k}(p)$ and $s \prec_{k} t$, $\left(\pi_{s t}(\bar{r}), t\right) \in T^{-k}(p)$ (so that Lemma4.1 is satisfied) and $\|(\bar{r}, s)\| \leq\left\|\left(\pi_{s t}(\bar{r}), t\right)\right\|$ (so that the construction can continue).

For any finite sequences $\bar{r}=\left(r_{1}, \ldots, r_{n}\right)$ and $\bar{s}=\left(s_{1}, \ldots, s_{m}\right)$, let $\left.\bar{r}\right\urcorner \bar{s}$ be the concatenation $\left.\left(r_{1}, \ldots, r_{n}, s_{1}, \ldots, s_{m}\right)\right)$. If $\bar{s}=\left(s_{1}\right)$, we may write $\bar{r} \frown s_{1}$ instead of $\bar{r}^{\frown}\left(s_{1}\right)$.

Let $\kappa=|X|$. Let $\{p\}=Z_{0}$, where 0 here denotes the one-point graph which is trivially augmented. Now consider $T^{-1}(p)$. Let $\tau_{1}=\left\{t_{\alpha}: \alpha \in \kappa\right\}$ be the augmented graph with order $t_{\alpha} \prec_{1} t_{\beta}$ if and only if $\alpha \neq 0=\beta$ (so that $t_{\tau_{1}}=t_{0}$ ), $n(t)=1$ for all $t \in \tau_{1}$, and $\pi_{s t}$ is the identity on $\mathbb{C}$. We identify $T^{-1}(p)$ with a subset of $Z_{\tau_{1}}$ as follows. Let $D_{p}$ be the set furnished by the statement of the lemma with the property that for each $z \in T^{-1}(p)$, there is some $y_{p z} \in D_{p}$ such that $\|z\| \leq\left\|y_{p z}\right\|$. For each $z \in T^{-1}(p)-D_{p}$, fix such a $y_{p z}$. By Lemma 3.2, there is an order-preserving map $o_{p z}$ from $\bigcup_{0 \leq k} T^{-k}(z)$ to $\bigcup_{0 \leq k} T^{-k}\left(y_{p z}\right)$. Identify each $y \in D_{p}$ uniquely with a point $\left(r_{y}, t_{0}\right) \in Z_{\tau_{1}}$, where $r_{y} \in \mathbb{C}$, and identify each $z \in T^{-1}(p)-D_{p}$ uniquely with $\left(r_{y_{p z}}, t_{\alpha}\right)$ for some $0<\alpha \in \kappa$. By Lemma 4.1 $T^{-1}(p)$, regarded as a subspace of $Z_{\tau_{1}}$, is Lindelöf, since for each $z=\left(r, t_{\alpha}\right) \in T^{-1}(p),\left(\pi_{t_{\alpha} t_{0}}(r), t_{0}\right)=\left(r, t_{0}\right)$ is in $T^{-1}(p)$. Notice also that $\|z\| \leq\left\|\left(\pi_{t_{\alpha}, t_{0}}(r), t_{0}\right)\right\|$. Clearly the restriction of the map $T$ from $T^{-1}(p)$ to $\{p\}$ is continuous. Moreover $m_{\tau_{1}}=2$.

Suppose now that, for $k>1$, we have embedded $T^{-k}(p)$ as a subset of $Z_{\tau_{k}}$ for some augmented graph $\tau_{k}$ with $m_{\tau_{k}}=k+1$ in such a way that for any $s \prec_{k} t \in \tau_{k}$ and any point $(\bar{r}, s) \in T^{-k}(p),\left(\pi_{s t}(\bar{r}), t\right) \in T^{-k}(p),\|(\bar{r}, s)\| \leq\left\|\left(\pi_{s t}(\bar{r}), t\right)\right\|$ and the restriction of $T$ to $T^{-k}(p)$ is a continuous function to $T^{-k+1}(p)$.

We define a new augmented graph $\tau_{k+1}$ from $\tau_{k}$. Let $\tau_{k+1}=\tau_{k} \times \kappa$ and define $(s, \alpha) \prec_{k+1}(t, \beta)$ if and only if either $s \prec_{k} t$ and $\beta=0$ or $s=t, \alpha \neq 0=\beta$. (Diagrammatically, to obtain $\tau_{k+1}$ from $\tau_{k}$, relabel each node $s \in \tau$ as $(s, 0)$ and then add new nodes $(s, \alpha), \alpha \in \kappa$, below the node $(s, 0)$.) Then $t_{\tau_{k+1}}=\left(t_{\tau}, 0\right)$ is the top element of $\tau_{k+1}$ and every branch of $\tau_{k+1}$ has length at most $m_{\tau_{k}}+1=k+2$. For each $(s, \alpha) \in \tau_{k+1}$, let $\#(s, \alpha)$ denote the number of elements of $\left\{(t, \beta):(s, \alpha) \preceq_{k}\right.$ $(t, \beta)\}$ for which $\beta=0$ and define $n(s, \alpha)=n(s)+\#(s, \alpha)$. Clearly $\#(s, \alpha) \leq m_{\tau_{k+1}}$, so that $n\left(t_{\tau_{k+1}}\right) \leq n\left(t_{\tau_{k}}\right)+m_{\tau_{k+1}}$ and $n(t, \beta) \leq n(s, \alpha)$, whenever $(s, \alpha) \prec_{k+1}(t, \beta)$.

Notice also that if $(s, \alpha) \prec(t, \beta)$ is the immediate $\prec_{k+1}$-predecessor of $(t, \beta)$, then either

(1) $s=t$ and $\alpha \neq 0=\beta$, in which case $n(s, \beta)=n(t, \alpha)$, or

(2) $s$ is the immediate $\prec_{k}$-predecessor of $t$ and $\alpha=\beta=0$, in which case $\#(s, \alpha)=\#(t, \beta)+1$ and $n(s, \alpha)=n(t, \beta)+1=n(t)+\#(t, \beta)+1$. 
In Case (1), define $\pi_{(s, \alpha)(t, \beta)}$ to be the identity from $\mathbb{C}^{n(s, \alpha)}=\mathbb{C}^{n(t, \beta)}$ to itself. In Case (2), define $\pi_{(s, \alpha)(t, \beta)}: \mathbb{C}^{n(s, \alpha)} \rightarrow \mathbb{C}^{n(t, \beta)}$ by

$$
\begin{aligned}
\pi_{(s, \alpha)(t, \beta)}\left(r_{1}, \ldots, r_{n(s)}, r_{n(s)+1}, \ldots, r_{n(s)+\#(s, \alpha)}\right) \\
=\pi_{s t}\left(r_{1}, \ldots, r_{n(s)}\right)^{\frown}\left(r_{n(s)+1}, \ldots, r_{n(s)+\#(t, \beta)}\right) .
\end{aligned}
$$

So the image of a point $\bar{r} \in C^{n(s \alpha)}$ under $\pi_{(s, \alpha)(t, \beta)}$ consists of the image of the first $n(s)$ coordinates under the map $\pi_{s t}$ followed by the first \# $(s, \alpha)-1=\#(t, \beta)$ coordinates of the remaining \# $\#(s, \alpha)$ coordinates of $\bar{r}$. Since there is a finite sequence of immediate predecessors between any $(s, \alpha) \prec_{k+1}(t, \beta)$, we can define $\pi_{(s, \alpha)(t, \beta)}$ by composing a finite number of such maps.

Claim. The map

$$
\begin{aligned}
& \Pi: Z_{\tau_{k+1}} \rightarrow Z_{\tau_{k}} \\
& \quad\left(\left(r_{1}, \ldots, r_{n(t, \alpha)}\right),(t, \alpha)\right) \mapsto\left(\left(r_{1}, \ldots, r_{n(t)}\right), t\right)
\end{aligned}
$$

is continuous

Proof. Recall that for $s \in \tau_{k}, B_{s}=\bigcup_{t \preceq_{k} s} \mathbb{C}^{n(t)} \times\{t\}$ and that for $\bar{r} \in \mathbb{C}^{n}$ and $j \in \mathbb{N}, B_{j}(\bar{r})$ is the $1 / 2^{j}$-ball about $\bar{r}$. For any $(\bar{r}, t) \in Z_{\tau_{k}}, j \in \mathbb{N}$ and finite $F \subseteq\left\{s \in \tau_{k}: s \prec_{k} t\right\}$,

$$
B(\bar{r}, t, j, F)=\left(\left(B_{j}(\bar{r}) \times\{t\}\right) \cup \bigcup_{s \prec_{k} t} \pi_{s t}^{-1}\left(B_{j}(\bar{r})\right) \times\{s\}\right) \backslash\left(\bigcup_{s \in F} B_{s}\right)
$$

is a basic open set in $Z_{\tau_{k}}$. Note from (1) above that $n(s, \alpha)=n(s, 0)$ for all $s \prec_{k} t$.

Now $\Pi^{-1}\left(B_{j}(\bar{r}) \times\{t\}\right)=\bigcup_{\alpha \in \kappa} B_{j}(\bar{r}) \times \mathbb{C}^{n(t, 0)-n(t)} \times\{(t, \alpha)\}$. Moreover, if $s \prec_{k} t$, then $\pi_{s t}\left(B_{j}(\bar{r})\right)=B_{j}(\bar{r}) \times \mathbb{C}^{n(s)-n(t)}$ so that $\Pi^{-1}\left(\pi_{s t}^{-1}\left(B_{j}(\bar{r})\right) \times\{s\}\right)=$ $\bigcup_{\alpha \in \kappa} B_{j}(\bar{r}) \times \mathbb{C}^{n(s, 0)-n(t)} \times\{(s, \alpha)\}$. By the definition of $\prec_{k+1}$, though, $\{(t, \alpha)$ : $\alpha \in \kappa\} \cup\left\{(s, \alpha): \alpha \in \kappa, s \prec_{k} t\right\}=\{(t, 0)\} \cup\left\{(s, \beta):(s, \beta) \prec_{k+1}(t, 0)\right\}$. It follows that

$$
\begin{aligned}
\Pi^{-1} & \left(\left(B_{j}(\bar{r}) \times\{t\}\right) \cup \bigcup_{s \prec_{k} t} \pi_{s t}^{-1}\left(B_{j}(\bar{r})\right) \times\{s\}\right) \\
= & \left(B_{j}(\bar{r}) \times \mathbb{C}^{n(t, 0)-n(t)} \times\{(t, 0)\}\right) \\
& \cup \underset{(s, \alpha) \prec_{k+1}(t, 0)}{\bigcup} \pi_{(s, \alpha)(t, 0)}^{-1}\left(B_{j}(\bar{r}) \times \mathbb{C}^{n(t, 0)-n(t)}\right) \times\{(s, \alpha)\} .
\end{aligned}
$$

Also

$$
\begin{aligned}
\Pi^{-1}\left(B_{s}\right) & =\bigcup_{u \preceq k} \Pi^{-1}\left(\mathbb{C}^{n(u)} \times\{u\}\right) \\
& =\bigcup_{u \preceq k} \bigcup_{\alpha \in \kappa} \mathbb{C}^{n(u, \alpha)} \times\{(u, \alpha)\} \\
& =\bigcup_{(u, \alpha) \preceq_{k+1}(s, 0)} \mathbb{C}^{n(u, \alpha)} \times\{(u, \alpha)\},
\end{aligned}
$$

which implies that $\Pi^{-1}\left(\bigcup_{s \in F} B_{s}\right)=\bigcup_{(s, 0) \in F \times\{0\}} B_{(s, 0)}$. It follows that the set $\Pi^{-1}(B(\bar{r}, t, j, F))$ is open in $Z_{\tau_{k+1}}$. 
It remains to embed $T^{-(k+1)}(p)$ into $Z_{\tau_{k+1}}$. Consider first a point $x=\left(\bar{r}, t_{\tau_{k}}\right) \in$ $T^{-k}(p)$. Let $D_{x}$ be the set furnished by the statement of the lemma with the property that for each $z \in T^{-1}(x)$ there is some $y_{x z} \in D_{x}$ such that $\|z\| \leq\left\|y_{x z}\right\|$. For each $z \in T^{-1}(x)-D_{x}$, fix such a $y_{x z}$. For each $y \in D_{x}$, pick a unique $r_{y} \in \mathbb{C}$ and identify $y$ with the point $\left(\bar{r}^{\frown} r_{y},\left(t_{\tau_{k}}, 0\right)\right)=\left(\bar{r}^{\frown} r_{y}, \tau_{\tau_{k+1}}\right)$. Identify each $z \in T^{-1}(x)-D_{x}$ uniquely with $\left(\bar{r}^{\frown} r_{y_{x z}},\left(t_{\tau_{k}}, \alpha\right)\right)$ for some $\alpha \in \kappa$.

Now suppose that $x_{i}=\left(\bar{r}_{i}, t_{i}\right)$ is a sequence of points from $T^{-k}(p)$, for each $0 \leq i \leq m$, such that

(1) $t_{0}=t_{\tau_{k}}$,

(2) $t_{i+1}$ is the immediate $\prec_{k}$-predecessor of $t_{i}$,

(3) $\pi_{t_{i+1} t_{i}}\left(\bar{r}_{i+1}\right)=\bar{r}_{i}$,

(4) the set $T^{-1}\left(x_{i}\right)$ has been embedded into $Z_{\tau_{k+1}}$ for each $i<m$,

(5) for each $i<m$, the set $D_{x_{i}}$ has been identified with a subset of $\mathbb{C}^{n\left(t_{i}, 0\right)} \times$ $\left\{t_{i}, 0\right\}$ in $Z_{\tau_{k+1}}$.

We know that $\left\|x_{m}\right\| \leq\left\|x_{m-1}\right\|$, which implies that there is an order-preserving map $o$ from $\bigcup_{j \geq 0} T^{j}\left(x_{m}\right)$ to $\bigcup_{j \geq 0} T^{j}\left(x_{m-1}\right)$ that, in particular, maps $T^{-1}\left(x_{m}\right)$ to $T^{-1}\left(x_{m-1}\right)$. For any $y \in D_{x_{m}}$, then, $o(y)=\left(\bar{r},\left(t_{m-1}, \alpha\right)\right) \in T^{-1}\left(x_{m-1}\right)$. By construction (as $\pi_{\left(t_{m-1}, \alpha\right)\left(t_{m-1}, 0\right)}$ is the identity), $\left(\pi_{\left(t_{m-1}, \alpha\right)\left(t_{m-1}, 0\right)}(\bar{r}),\left(t_{m-1}, 0\right)\right)=$ $\left(\bar{r},\left(t_{m-1}, 0\right)\right) \in D_{x_{m-1}}$ and $\left\|\left(\bar{r},\left(t_{m-1}, \alpha\right)\right)\right\| \leq\left\|\left(\bar{r},\left(t_{m-1}, 0\right)\right)\right\|$, so that $\|y\| \leq$ $\left\|\left(\bar{r},\left(t_{m-1}, 0\right)\right)\right\|$. Hence for each $y \in D_{x_{m}}$, we can fix some $w_{y}=\left(\bar{r}_{y},\left(t_{m-1}, 0\right)_{y}\right) \in$ $D_{x_{m-1}}$ such that $\|y\| \leq\left\|w_{y}\right\|$. Since $\left|D_{x_{m}}\right| \leq \mathfrak{c}$, we can associate a unique $r_{w y} \in \mathbb{C}$ to each $y$ and $w$ for which $w_{y}=w$. Given $y \in D_{x_{m}}$ and $w_{y}=$ $\left(\bar{r}_{y},\left(t_{m-1}, 0\right)_{y}\right) \in D_{x_{m-1}}$, identify $y$ with the point $\left(\bar{r}_{y}{ }^{\wedge} r_{w y},\left(t_{m}, 0\right)\right)$. Now for each $z \in T^{-1}\left(x_{m}\right)-D_{x_{m}}$, fix some $y_{x_{m} z}=\left(\bar{r}_{x_{m} z},\left(t_{m}, 0\right)\right) \in D_{x_{m}}$ such that $\|z\| \leq\left\|y_{x_{m}}\right\|$ and associate $z$ uniquely with $\left(\bar{r}_{x_{m} z},\left(t_{m}, \alpha\right)\right)$ for some $\alpha \in \kappa$. This embedding ensures that for any $s \prec_{k+1} t \in \tau_{k+1}$ and any point $(\bar{r}, s) \in T^{-k-1}(p)$, $\left(\pi_{s t}(\bar{r}), t\right) \in T^{-k-1}(p),\|(\bar{r}, s)\| \leq\left\|\left(\pi_{s t}(\bar{r}), t\right)\right\|$. Moreover, by construction, for any $(\bar{r}, t) \in Z_{\tau_{k+1}}, T(\bar{r}, t)=\Pi(\bar{r}, t)$ so that $T \uparrow_{T^{-k-1}(p)}$ is continuous as required.

Exactly the same argument can be used to prove the following lemma dealing with $\mathbb{N}$-orbits. We recall that $T^{-0}(x)=\{x\}$ and note that the index $\alpha$ is introduced here purely for notational consistency in Section 5 .

Lemma 4.3. Let $T: X \rightarrow X$ and let $N_{\alpha}$ be an $\mathbb{N}$-orbit of $T$ with spine $\left\{x_{\alpha, n}: 0 \leq n\right\}$ chosen so that $T^{-1}\left(x_{\alpha}, 0\right)=\emptyset$. Let $S_{\alpha, 0,0}=\left\{x_{\alpha, 0}\right\}$ and $S_{\alpha, 0, k}=\emptyset$ for all $0<k$, and let

$$
S_{\alpha, n, k}= \begin{cases}\left\{x_{\alpha, n}\right\} & k=0, \\ T^{-k}\left(x_{\alpha, n}\right) \backslash T^{-(k-1)}\left(x_{\alpha, n-1}\right) & 0<k,\end{cases}
$$

for all $0<n$. Suppose that, for every $x \in N_{\alpha}$, there is a subset $D_{x} \subseteq T^{-1}(x)$ such that

(1) $\left|D_{x}\right| \leq \mathfrak{c}$ and

(2) for each $z \in T^{-1}(x)$, there is some $y_{x z} \in D_{x}$ such that $\|z\| \leq\left\|y_{x z}\right\|$.

Then for each $n$ and $k$ in $\mathbb{N}$, there is a (zero-dimensional) Tychonoff Lindelöf topology $\mathcal{T}_{\alpha, n, k}$ on $S_{\alpha, n, k}$ with respect to which the action of $T$ from $S_{\alpha, n, k+1}$ to $S_{\alpha, n, k}$ is continuous.

Proof. Note that $N_{\alpha}=\bigcup_{n, k \in \mathbb{N}} S_{\alpha, n, k}$. Since $N_{\alpha}$ is an $\mathbb{N}$-orbit, by Lemma 3.3, $\|x\|<\mathfrak{c}^{+}$for every $x \in N_{\alpha}$ and, moreover, for each $0<n$, we can choose $D_{x_{\alpha, n}}$ so 
that it does not contain $x_{\alpha, n-1}$. We can, therefore, apply the proof of Lemma 4.2 to the restriction of $T$ to $\left(\bigcup_{0<k} T^{-k}\left(x_{\alpha, n}\right)\right) \backslash\left(\bigcup_{0<k} T^{-k}\left(x_{\alpha, n-1}\right)\right)=\bigcup_{0<k} S_{\alpha, n, k}$ with $p=x_{\alpha, n}$.

\section{COMBINING ORBits}

In this section we complete the proof of (1) of Theorem 1.6. Given the constructions of Section 4 we show that there is a Lindelöf topology on $X$ provided $T^{\mathfrak{c}^{+}}(X)=T^{\mathfrak{c}^{+}+1}(X) \neq \emptyset$. In this situation, (3) of Lemma 3.3 ensures that there are $\mathbb{Z}$-orbits or $n$-cycles. We prove the second statement of Theorem 1.6 that there is a zero-dimensional, Lindelöf topology on $X$ if $T^{\alpha}(X)=\emptyset$ for some $\alpha \in \mathfrak{c}^{+}$, which implies that $X$ consists solely of $\mathbb{N}$-orbits, using a modification of the proof of Lemma 4.2 .

Proof of Theorem 1.6 (1). By Lemma 3.3 (3), we know that $X$ has some combination of $\mathbb{Z}$-orbits and $n$-cycles. Since a free union of countably many Lindelöf spaces is again Lindelöf, we may assume, without loss of generality, that either (a) $X$ consists exclusively of $\mathbb{Z}$-orbits and $\mathbb{N}$-orbits or (b) $X$ consists of $m$-cycles, for some fixed $m \in \mathbb{N}$, and $\mathbb{N}$-orbits. In fact it is sufficient to consider the following four cases:

(ai) $X$ consists entirely of $\mathbb{Z}$-orbits,

(aii) $X$ has one $\mathbb{Z}$-orbit and all other orbits are $\mathbb{N}$-orbits,

(bi) $X$ consists entirely of $m$-cycles for some fixed $m \in \mathbb{N}$,

(bii) $X$ has one $m$-cycle and all other orbits are $\mathbb{N}$-orbits.

Case (a): Let us assume that we have chosen appropriate spines for each orbit. Index the $\mathbb{Z}$-orbits of $T$ as $\left\{Z_{\alpha}: \alpha \in \zeta\right\}$ and denote the spine points of $Z_{\alpha}$ by $\left\{z_{\alpha, n}: n \in \mathbb{Z}, \alpha \in \zeta\right\}$ so that $T\left(z_{\alpha, n+1}\right)=z_{\alpha, n}$. Index the $\mathbb{N}$-orbits of $T$ by $\left\{N_{\alpha}: \alpha \in \nu\right\}$ and denote the spine points of $N_{\alpha}$ by $\left\{x_{\alpha, n}: n \in \mathbb{N}, \alpha \in \nu\right\}$ so that $T\left(x_{\alpha, n+1}\right)=x_{\alpha, n}$ and $T^{-1}\left(x_{0}\right)=\emptyset$.

By Lemmas 3.3 and 4.2 , for each non-spine point $p \in T^{-1}\left(z_{\alpha, n}\right)$ and for each $k \geq 0$ there is a (zero-dimensional) Lindelöf topology $\mathcal{T}_{p, k}$ on $T^{-k}(p)$ with respect to which the action of $T$ from $T^{-k-1}(p)$ to $T^{-k}(p)$ is continuous. Recall that we let $T^{-0}(x)=\{x\}$ and $\mathcal{T}_{x, 0}=\{\emptyset,\{x\}\}$. Define $S_{\alpha, l, k}=\left\{x_{\alpha, l}\right\}$ if $k=0$, and $S_{\alpha, l, k}=T^{-k}\left(x_{\alpha, l}\right) \backslash T^{-(k-1)}\left(x_{\alpha, l-1}\right)$ if $0<k$. Since every $x \in X$ with $\|x\|<\infty$ has rank $\|x\|<\mathfrak{c}^{+}$, Lemmas 3.3 and 4.3 imply that, for each $\alpha \in \nu$ and each $k$ and $l$ in $\mathbb{N}$, there is a zero-dimensional, Lindelöf topology $\mathcal{T}_{\alpha, l, k}$ on $S_{\alpha, l, k}$ with respect to which the action of $T$ from $S_{\alpha, l, k+1}$ to $S_{\alpha, l, k}$ is continuous. Again $T^{-0}\left(x_{\alpha, l}\right)=\left\{x_{\alpha, l}\right\}$ and $\mathcal{T}_{\alpha, l, 0}=\left\{\emptyset,\left\{x_{\alpha, l}\right\}\right\}$.

Case (ai): $X$ consists entirely of $\mathbb{Z}$-orbits. For each $\alpha \in \lambda$ and $n \in \mathbb{Z}$, index the non-spine points of $T^{-1}\left(z_{\alpha, n+1}\right)-\left\{z_{\alpha, n}\right\}$ by $\left\{p_{\alpha, n, \beta}: \beta \in \mu_{\alpha, n+1}\right\}$. Notice that for any $n \in \mathbb{Z}$, any $k>0$ and any $q \in T^{-(k-1)}\left(p_{\alpha, n+k, \beta}\right)$, we have $T\left(p_{\alpha, n, \beta}\right)=z_{\alpha, n+1}$ and $z_{\alpha, n+k}=T^{k}\left(z_{\alpha, n}\right)=T^{k}(q)$. For each $\alpha \in \zeta$ and $n \in \mathbb{Z}$, let

$$
L_{\alpha, n}=\bigcup_{0 \leq k} T^{-k}\left(T^{k}\left(z_{\alpha, n}\right)\right)=\left\{z_{\alpha, n}\right\} \cup \bigcup\left\{T^{-(k-1)}\left(p_{\alpha, n+k, \beta}\right): 0<k \beta \in \mu_{\alpha, n+k}\right\} .
$$

Notice that $Z_{\alpha}=\bigcup_{n \in \mathbb{Z}} L_{\alpha, n}$ and that, for all $n \in \mathbb{Z}, T^{-1}\left(L_{\alpha, n}\right)=L_{\alpha, n-1}$ and $T\left(L_{\alpha, n}\right) \subset L_{\alpha, n+1}$ (and $L_{\alpha, n+1}-T\left(L_{\alpha, n}\right)$ is exactly the set of points $p \in L_{\alpha, n+1}$ for which $\left.T^{-1}(p)=\emptyset\right)$. Moreover, if $L_{n}=\bigcup_{\alpha \in \zeta} L_{\alpha, n}$, then $X=\bigcup_{n \in Z} L_{n}$.

Topologize $X$ as follows: 
(1) for each $\alpha \in \zeta, n \in \mathbb{Z}, \beta \in \mu_{\alpha, n}$ and $k \geq 0$, let $T^{-k}\left(p_{\alpha, n, \beta}\right)$ be a clopen set with relative topology $\mathcal{T}_{p_{\alpha, n, \beta}, k}$ so that each point $p \in T^{-1}\left(z_{n}\right)-\left\{z_{n-1}\right\}$ is isolated;

(2) for each $\alpha>0$ and $n \in \mathbb{Z}$, let basic open neighbourhoods about the point $z_{\alpha, n}$ take the form

$$
L_{\alpha, n}-\bigcup\left\{T^{-(k-1)}\left(p_{\alpha, n+k, \beta}\right):(k, \beta) \in F\right\},
$$

for some finite set $F$;

(3) for each $n \in \mathbb{Z}$, let basic neighbourhoods of $z_{0, n}$ take the form

$$
\left(L_{0, n}-\bigcup\left\{T^{-(k-1)}\left(p_{0, n+k, \beta}\right):(k, \beta) \in F\right\}\right) \cup \bigcup\left\{L_{\alpha, n}: 0<\alpha \in \zeta, \alpha \notin G\right\},
$$

for finite sets $F$ and $G$.

Clearly every point of $X$ has a clopen neighbourhood in this topology so that $X$ is zero-dimensional and Tychonoff. To see that $X$ is Lindelöf, it is enough to note that, for each $n \in \mathbb{Z}, L_{n}$ is Lindelöf. But if $\mathcal{U}$ is any open cover of $L_{n}$ and $z_{0, n} \in U \in \mathcal{U}$, then $L_{n} \backslash U$ is a subset of $\bigcup_{\alpha \in G} L_{\alpha, n} \cup \bigcup\left\{T^{-(k-1)}\left(p_{0, n+k, \beta}\right):(k, \beta) \in F\right\}$ for some finite $F$ and $G$. If $z_{\alpha, n} \in U_{\alpha} \in \mathcal{U}$ for any $\alpha \in G$, then $L_{\alpha, n} \backslash U_{\alpha}$ is a subset of $\bigcup\left\{T^{-(k-1)}\left(p_{\alpha, n+k, \beta}\right):(k, \beta) \in F_{\alpha}\right\}$. Hence $L_{n}-\left(U \cup \bigcup_{\alpha \in G} U_{\alpha}\right)$ is covered by finitely many sets of the form $T^{j}(p)$, all of which are clopen and Lindelöf.

Continuity of $T$ follows directly, since the inverse image of a basic open set under $T$ is again a basic open set.

Case (aii): $X$ consists of exactly one $\mathbb{Z}$-orbit and $\mathbb{N}$-orbits. Let the $\mathbb{Z}$-orbit be $Z_{0}$, and as in case (ai) let $\left\{p_{0, n, \beta}: \beta \in \mu_{\alpha, n}\right\}$ denote the points of $T^{-1}\left(z_{0, n+1}\right)-\left\{z_{o, n}\right\}$ and let $L_{0, n}=\bigcup_{0 \leq k} T^{-k}\left(T^{k}\left(z_{0, n}\right)\right)$. Let $X$ have the topology generated by the following sets:

(1) for each $\alpha \in \nu, k$ and $l$ in $\mathbb{N}$, let $S_{\alpha, l, k}$ be a clopen set with relative topology $\mathcal{T}_{\alpha, l, k}$

(2) for each $n \in \mathbb{Z}$, let basic open neighbourhoods of $z_{0, n}$ take the form

$$
\begin{aligned}
& \left(L_{0, n}-\bigcup\left\{T^{-(k-1)}\left(p_{0, n+k, \beta}\right):(k, \beta) \in F\right\}\right) \\
& \quad \cup\left\{S_{\alpha, l, k}: \alpha \in \nu, k, l \in \mathbb{N}, l-k=n, \quad(\alpha, l, k) \notin G\right\},
\end{aligned}
$$

for finite sets $F$ and $G$.

Again it is clear that this topology on $X$ is zero-dimensional and Tychonoff. For each $n \in \mathbb{Z}$, let $L_{n}=L_{n, 0} \cup \bigcup\left\{S_{\alpha, l, k}: \alpha \in \nu, k, l \in \mathbb{N}, l-k=n\right\}$ so that $X=\bigcup_{n \in \mathbb{Z}} L_{n}$. Each $L_{n}$ is Lindelöf, since each $S_{\alpha, l, k}$ is Lindelöf. Hence $X$ is Lindelöf. Continuity again follows from the definition of the topology.

In Case (bi), $X$ consists solely of $m$-cycles for some $m \in \mathbb{N}$; in Case (bii), $X$ consists of a single $m$-cycle and $\mathbb{N}$-orbits. In both cases the proof is identical to that of Cases (ai) and (aii) except that the indexing number $n \in \mathbb{Z}$ is taken modulo $m$, so that, for example, $z_{\alpha, n}=z_{\alpha, n+m}$.

Proof of Theorem 1.6 (2). By Lemma 3.3 (4), $X$ consists entirely of $\mathbb{N}$-orbits. Let $\left\{N_{\alpha}: \alpha \in \nu\right\}$ list the $\mathbb{N}$-orbits and let $\left\{x_{\alpha, n}: 0 \leq n\right\}$ index the spine of $N_{\alpha}$ so that $T\left(x_{n}\right)=x_{n+1}$ and $T^{-1}\left(x_{0}\right)=\emptyset$. Since $\|x\|<\mathfrak{c}^{+}$, for all $x \in X$, 3.3 (1) implies that for each $x \in X$ there is a subset $D_{x} \subseteq T^{-1}(x)$ such that $\left|D_{x}\right| \leq \mathfrak{c}$ and, for each $z \in T^{-1}(x)$, there is some $y_{z} \in D_{x}$ such that $\|z\| \leq\left\|y_{z}\right\|$. Moreover, by 
Lemma 3.3 (2), we may assume that for all $\alpha \in \nu$ and all $n \in \mathbb{N}, x_{\alpha, n} \notin D_{x_{\alpha, n+1}}$. Now, since the cardinality of the set $\left\{\left\|x_{\alpha, n}\right\|: \alpha \in \nu, n \in \mathbb{N}\right\}$ is at most $\mathfrak{c}$, there is a subset $D \subseteq \nu$ such that

(1) $|D| \leq \mathfrak{c}$ and

(2) for all $\alpha \in \nu$, there is some $\eta \in D$ such that $\left\|x_{\alpha, n}\right\| \leq\left\|x_{\eta, n}\right\|$ for all $n \in \mathbb{N}$.

As before, let $S_{\alpha, 0,0}=\left\{x_{0}\right\}$ and $S_{\alpha, 0, k}=\emptyset$ for all $0<k$, and let

$$
S_{\alpha, n, k}= \begin{cases}\left\{x_{\alpha, n}\right\} & k=0, \\ T^{-k}\left(x_{\alpha, n}\right) \backslash T^{-(k-1)}\left(x_{\alpha, n-1}\right) & 0<k,\end{cases}
$$

for all $0<n$.

Let $X^{*}=X \cup\left\{p_{n, i}: i=0,1, n \in \mathbb{N}\right\}$, where $p_{n, i} \notin X$ for any $n \in \mathbb{N}$ and $i=0,1$. We shall define a map $T^{*}: X^{*} \rightarrow X^{*}$ and a topology on $X^{*}$ with respect to which $T^{*}$ is continuous from which we define a Lindelöf topology on $X$ with respect to which $T$ is continuous. For all $n \in \mathbb{N}$ and $\alpha \in \nu$ define

$$
T^{*}(x)= \begin{cases}p_{n, 0} & \text { if } x=p_{n, 0}, \\ p_{n, 0} & \text { if } x=p_{n, 1}, \\ p_{n, 1} & \text { if } x=x_{\alpha, n} \\ T(x) & \text { if } x \in \bigcup_{0<k} S_{\alpha, n, k} .\end{cases}
$$

Note that $T^{*}$ is a function with countably many 1-cycles, namely $\bigcup_{0 \leq n} T^{*-1}\left(p_{n, 0}\right)$, for each $n \in \mathbb{N}$, each with spine point $p_{n, 0}$ such that $T^{*-1}\left(p_{n, 0}\right)=\left\{p_{n, 0}, p_{n, 1}\right\}$ and $T^{*-1}\left(p_{n, 1}\right)=\left\{x_{\alpha, n}: \alpha \in \nu\right\}$. For each non-spine point $x$ of each 1-cycle let

$$
D_{x}^{*}= \begin{cases}D_{x} & \text { if } p_{n, 1} \neq x \in X, \\ \left\{x_{\eta, n}: \eta \in D\right\} & \text { if } x=p_{n, 1} .\end{cases}
$$

It follows that the action of $T^{*}$ on $\bigcup_{k \in \mathbb{N}} T^{*-k}\left(p_{n, 1}\right)$ satisfies the conditions of Lemma 4.2, so that for each $n$ and $k$ in $\mathbb{N}$ there is a zero-dimensional Lindelöf topology $\mathcal{T}_{n, k}$ on $T^{*-k}\left(p_{n, 1}\right)$ with respect to which the action of $T^{*}$ is continuous.

Now that $X=\bigcup_{n \in \mathbb{N}} \bigcup_{0<k} T^{*-k}\left(p_{n, 1}\right)$ and since $T^{*-k}\left(p_{n, 1}\right)$ is zero-dimensional and Lindelöf, there is a zero-dimensional, Lindelöf $\mathcal{T}$ on $X$ defined by declaring each $T^{*-k}\left(p_{n, 1}\right)$ to be clopen with relative topology $\mathcal{T}_{n, k}$. It remains to ensure that the action of $T$ with respect to this topology is continuous. So let $U$ be an open subset of some clopen set $T^{*-k}\left(p_{n, 1}\right)$. If $1<k$, then $T^{*-1}(U)=T^{-1}(U)$, so that $T^{-1}(U)$ is open. If $k=1$, then $T^{-1}(U)=T^{*-1}(U) \cup\left\{x_{\alpha, n-1}: x_{\alpha, n} \in U\right\}$, which is open provided $\left\{x_{\alpha, n-1}: x_{\alpha, n} \in U\right\}$ is open in $T^{*-1}\left(p_{n-1,1}\right)$. But this is easily arranged. Associate $T^{*-1}\left(p_{0,1}\right)$ with a subset of $Z_{\tau_{1}}$ as in the proof of Lemma 4.2 so that $x_{\alpha, 0}$ is identified with the point $\left(r_{\alpha}, t_{\alpha}\right) \in Z_{\tau_{1}}$. By the choice of the set $D$, for all $\alpha \in \nu$ there is $\eta \in D$ such that $\left\|x_{\alpha, n}\right\| \leq\left\|x_{\eta, n}\right\|$, for all $n \in \mathbb{N}$. Since $T\left(x_{\alpha, n}\right)=x_{\alpha, n+1}$ for all $\alpha \in \nu$ and $n \in \mathbb{N}, D_{p_{n+1,1}}^{*}=T\left(D_{p_{n, 1}}\right)$. Therefore, we can embed each $T^{*-1}\left(p_{n, 1}\right)$ as a subspace of the space $Z_{\tau_{1}}$ simply by identifying $x_{\alpha, n}$ with the point $\left(r_{\alpha}, t_{\alpha}\right)$. The construction of Lemma 4.2 is unaffected and we are done, as, for each $n \in \mathbb{N}$, $T$ is a homeomorphism from $\left\{x_{\alpha, n}: \alpha \in \nu\right\}$ to $\left\{x_{\alpha, n+1}: \alpha \in \nu\right\}$.

Proof of Corollary 1.7. For (1), if $T$ is a surjection, then (1) of Theorem 1.6 obviously holds. For (2) and (3), if $T$ is an injection, then certainly it is $<\mathfrak{c}$-to- 1 , and if it is $<\mathfrak{c}$-to-one, then, for each $x \in X$, either $\|x\|=\infty$ or $\|x\|<\mathfrak{c}$, so that either (1) or (2) of Theorem 1.6 holds. For (4), if $T$ is $\leq \mathfrak{c}$-to-one, then, for each $x \in X$, 
either $\|x\|=\infty$ or $\|x\|<\mathfrak{c}^{+}$, which implies that $T^{\mathfrak{c}^{+}}(X)=T^{\mathfrak{c}^{+}+1}(X)$. Since $T$ has at least one orbit that is not an $\mathbb{N}$-orbit, $T^{\mathfrak{c}^{+}}(X) \neq \emptyset$, and we can apply (1) of Theorem 1.6.

\section{REFERENCES}

[1] D. Ellis, Orbital topologies, Quart. J. Math. Oxford (2), 4 (1953), 117-119. MR0056281 $(15: 51 \mathrm{c})$

[2] R. Engelking, General topology, Sigma Series in Pure Mathematics, Vol. 6, Heldermann Verlag, Berlin, 1989. MR1039321 (91c:54001)

[3] C. Good, S. Greenwood, R. W. Knight, D. W. McIntyre, and S. Watson, Characterizing continuous functions on compact spaces, Adv. Math. 206 (2006), 695-728. MR2263719 (2007i:54027)

[4] J. de Groot and H. de Vries, Metrization of a set which is mapped into itself, Quart. J. Math. Oxford (2), 9 (1958), 144-148. MR0105664(21:4402)

[5] R. Hodel, Cardinal Functions. I, in K. Kunen and J. E. Vaughan, eds., Handbook of SetTheoretic Topology, North-Holland, Amsterdam, 1984. MR776620 (86j:54007)

[6] A. Iwanik, How restrictive is topological dynamics? Comment. Math. Univ. Carolin., 38 (1997), 563-569. MR1485077 (98k:54078)

[7] A. Iwanik, L. Janos and F. A. Smith, Compactification of a map which is mapped to itself, Proceedings of the Ninth Prague Topological Symposium (2001) (electronic), Topology Atlas, North Bay, ON, 2002, 165-169. MR1906837 (2003f:54052)

[8] L. Janos, An application of combinatorial techniques to a topological problem, Bull. Austral. Math. Soc., 9 (1973), 439-443. MR0339090 (49:3853)

[9] T. Jech, Set Theory, The Third Millennium Edition, Springer-Verlag, Berlin, 2003. MR1940513 (2004g:03071)

[10] K. Kunen, Set Theory, an Introduction to Independence Proofs, North-Holland, Amsterdam, 1980. MR597342 (82f:03001)

[11] H. de Vries, Compactification of a set which is mapped onto itself, Bull. Acad. Polonaise des Sci. Cl. III, 5 (1957), 943-945. MR0092144 (19:1069f)

School of Mathematics, University of Birmingham, Birmingham B15 2Tt, United KINGDOM

E-mail address: c.good@bham.ac.uk

University of Auckland, Private Bag 92019, Auckland, New Zealand

E-mail address: sina@math.auckland.ac.nz 